\title{
CO-RS-COMPACT TOPOLOGIES
}

\author{
M. E. ABD EL-MONSEF, A. M. KOZAE AND A. A. ABO KHADRA
}

\begin{abstract}
A topology $R(\tau)$ is contructed from a given topolgy $\tau$ on a set $X . R(\tau)$ is coarser than $\tau$, and the following are some results based on this topology:

1. Continuity and RS-continuity are equivalent if the codomain is retopologized by $R(\tau)$.

2. The class of semi-open sets with respect to $R(\tau)$ is a topology.

3. $T_{2}$ and semi- $T_{2}$ properties are equivalent on a space whose topology is $R(\tau)$.

4. Minimal $R_{0}$-spaces are RS-compact:
\end{abstract}

\section{Introduction}

Throughout the present paper $(X, \sigma)$ and $(Y, \tau)$ are topological spaces on which no separation axioms are assumed unless explicitly stated. A set $S$ is said to be regular open (resp. regular closed) if $S=\operatorname{int}(\operatorname{cl}(S))$ (resp. $S=\operatorname{cl}(\operatorname{int}(S))$. A set $S$ is said to be $\alpha$-open [21] (resp. preopen [15]), if $S \subset \operatorname{int}($ cl int $(S)$ ), (resp. $S \subset$ int cl(S)). $A$ set $S$ is said to be regular semi-open [4] (resp. semi-open [13]) if there exists a regular open (resp. open) set 0 such that $0 \subset S \subset \operatorname{cl}(0)$. It should be noticed that the complement of a regular semi-open set is also regular semi-open. The family of all regular semi-open (resp. regular open, regular closed, $\alpha$-open, preopen and semi-open) sets in $X$ is denoted by $R S O(X)$ (resp.

Received May 5, 1992.

1991 AMS Subject Classification Codes: 54A10, 54C10, 54D10, 54D20.

Key words and phrases: RS-compact spaces, RS-continuous functions, extremely disconnected, semi- $T_{2}, \alpha$-compact spaces, $R_{0}$-spaces. 
$R O(X), R C(X), \alpha O(X), P O(X), S O(X))$. A space $X$ is said to be extremely disconnected if for every open set 0 of $X, \operatorname{cl}(0)$ is open in $X$.

In 1980, Hong [9] has introduced a new class of topological spaces called RS-compact spaces which are characterized by the following property "Every regular closed cover has a finite subfamily, the interiors of whose members cover X".

Note. The definition of RS-compact space in the sense of Hong is equivalent to that of an I-compact space in the sense of Cameron [5]. In 1985 Noiri, [22] has introudced RS-compact relative to $X$. "A subset $S$ of $X$ is RS-compact relative to $X$ if for every cover $\left\{V_{i}: i \in I\right\}$ of $S$ by regular closed sets of $X$, there exists a finite subset $I_{0}$ of $I$ such that $S \subset \cup\left\{\operatorname{int}\left(V_{i}\right): i \in I_{0}\right\}$. The relationship between RS-compacteness and ordinary compactness, with examples to illustrate that neither implies the other was studied in [22].

In 1989, Abd El-Monsef et al., [2] have introduced RS-continuous function "A function $f: X \rightarrow Y$ is called RS-continuous if for each $x \in X$ and each open set $V \subset Y$ containing $f(x)$ having RS-compact complement, there exists an open set $U \subset X$ containing $x$ such that $f(U) \subset V^{\prime \prime}$. A space $X$ is said to be almost normal space [17] if for every two disjoint regular closed subsets $F_{1}$ and $F_{2}$ of $X$, there exist two disjoint open sets $U$ and $V$ in $X$ such that $F_{1} \subset U$ and $F_{2} \subset V$. A space $X$ is semi- $T_{2}$ [14] (resp. semi- $T_{2}^{\prime}$ [1]) if for each $x, y \in X$, $x \neq y$, there exist $U$ and $V \in S O(X)$ such that $x \in U, y \in V$ and $U \cap V=\phi$ (resp. $\operatorname{cl}(U) \cap \operatorname{cl}(V)=\phi$ ). The space $(Y, \tau)$ is $R_{0}$ [6] if for each $G \in \tau, x \in G$ implies $c l\{x\} \subseteq G$. We observe that in every $R_{0}$ topological space the closure of a singleton set is compact. A space $X$ is $\alpha$-compact [3] (resp. strongly compact [16]) if each cover of $X$ by $\alpha$-open (resp. preopen) sets in $X$ has a finite subcover.

Theorem 1.1. [22]. If $a \in R S O(X)$ and $B$ is $R S$-compact relative to $X$, then $A \cap B$ is $R S$-compact relative to $X$.

Theorem 1.2. [22]. Let $A \in R O(X)$. Then $A$ is $R S$-compact iff $A$ is $R S$-compact relative to $X$. 
Theroem 1.3. [22]. If $X$ is $R S$-compact and $A \in R O(X)$, then $A$ is $R S$-compact.

Thereom 1.4. [22]. Let $X_{0}$ be an open set of $X$. Then we have:

(1) If $A \subset X_{0}$, then $\operatorname{Int}_{X}(A)=\operatorname{Int}_{X_{0}}(A)$.

(2) If $V \in R S O(X)$, then $V \cap X_{0} \in R S O\left(X_{0}\right)$.

Theorem 1.5. [5]. Any RS-compact is extremely disconnected.

Theorem 1.6. [10]. Every compact Hausdorff space is normal.

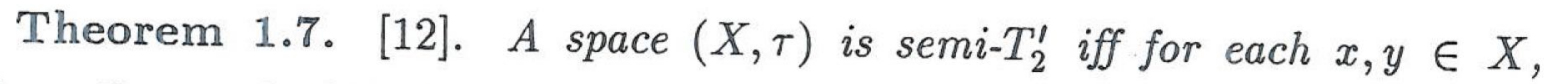
$x \neq y$, there exist $W_{1}, W_{2} \in R C(X, \tau)$ containing $x, y$ respectively, such that $W_{1} \cap W_{2}=\phi$.

Theorem 1.8. [11]. The following statements are equivalent for a space $(X, \tau)$ :

(a) $(X, \tau)$ is extremely disconnected.

(b) For each $A \in S O(X, \tau), \operatorname{cl}(A) \in \tau$.

(c) For each $A, B \in S O(X, \tau), \operatorname{cl}(A \cap B)=\operatorname{cl}(A) \cap \operatorname{cl}(B)$.

\section{CO-RS-Compact Topologies}

Let $(Y, \tau)$ be a topological space, and consider $R^{\prime}(\tau)=\{U \in \tau: Y-U$ is $R S$-compact relative to $\tau\} . R^{\prime}(\tau)$ is a base for a topology $R(\tau)$ on $Y$, called the $C O$ - $R S$-compact topology on $Y$. We shall denote by $(Y, R(\tau))$ to be a $C O-R S$ compact space of $(Y, \tau)$, and $c l_{R(\tau)}(S)$ (resp. $\operatorname{int}_{R(\tau)}(S)$ ) will denote the closure (resp. interior) with respect to $R(\tau)$ of a subset $S$ of $(Y, R(\tau))$. From definition we have $R(\tau) \subset \tau$, and the following lemma is a direct consequence.

Lemma 2.1. The function $f:(X, \sigma) \rightarrow(Y, \tau)$ is RS-continuous iff $f$ : $(X, \sigma) \rightarrow(Y, R(\tau))$ is continuous.

Theorem 2.1. For any topological space $(Y, \tau),(Y, R(\tau))$ is $R S$-compact 
space.

Proof. Consider the $R(\tau)$ regular closed cover $\triangle=\left\{V_{i}: i \in I\right\}$ of $Y$, and $V$ be some nonempty member of $\triangle$, there exists $R(\tau)$ open set $U$ such that $U \subset V \subset R-\operatorname{cl}(U)$, and $Y-U$ is $R S$-compact relative to $\tau$. By using Theorem 1.1 and Theorem 1.2. $(Y-V)$ is $R S$-compact subspace of $Y$. Assume that $A=Y-V$, thus $V_{i} \cap A \in R S O(A)$, for each $i \in I$ (By using Theorem 1.4), and $A=\cup\left\{V_{i} \cap A: i \in I\right\}$. There exists a finite subset $I_{0}$ of $I$ such that $A=\cup\left\{\operatorname{int}_{A}\left(V_{i} \cap A\right): i \in I_{0}\right\}$. From Theorem 1.4., we have $\operatorname{int}_{A}\left(V_{i} \cap A\right)=$ $\operatorname{int}_{Y}\left(V_{i} \cap A\right) \subset \operatorname{int}_{Y}\left(V_{i}\right)$ for each $i \in I_{0}$. Hence $A \subset U\left(\operatorname{int}_{Y}\left(V_{i}\right): i \in I_{0}\right\}$, and $A=Y-V$ is $R S$-compact relative to $R(\tau)$. Thus $(Y, R(\tau))$ is $R S$-compact space.

Remark 2.1. From Theorem 2.1, we notice that each topological space which contains at least a proper open set with $R S$-compact complement has a coarser extremely disconnected topology $R(\tau)$ [not indiscrete].

Proposition 2.1. The following statements are hold:

(a) $R O(Y, R(\tau)) \subset R O(Y, \tau)$.

(b) $c l_{\tau} G=c l_{R} G$, for all $G \in R O(Y, R(\tau))$.

(c) $R S O(Y, R(\tau) \subset R S O(Y, \tau)$.

Proof. (a) Let $G \in R O(Y, R(\tau))$, then $G=\operatorname{int}_{R} c l_{R}(G)=c l_{R}(G)[(Y, R(\tau))$ is extremely disconnected]. But $c l_{\tau} G \subset c l_{R} G$, implies that $G=c l_{\tau} G$ and $G=$ $\operatorname{int}_{\tau} c l_{\tau} G$.

(b) From (a), the proof is obvious.

(c) By using (b), the result follows.

Propsoition 2.2. Let $X$ be an extremely disconnected space then:

(a) The union of a finite regular open sets is a regular open set.

(b) If $X$ is the union of a finite number of regular open $R S$-compact subspaces, then $X$ is $R S$-compact space.

Lemma 2.2. If $(X, \tau)$ is extremely disconnected, then every regular closed 
subset is regular open.

Proof. Let $F \in R C(X, \tau)$, then $F=c l \operatorname{int} F=\operatorname{int} c l \operatorname{int} F[(X, \tau)$ is extremely disconnected]. Thus $c l F=c l \operatorname{int} F$, implies int $c l F=\operatorname{int} c l \operatorname{int} F=F$, and $F \in \mathbb{R} O(X, \tau)$.

Theorem 2.2. If $(Y, R(\tau))$ is almost normal space, then $(Y, \tau)$ is $R S$ compact.

\section{Proof. There are two cases:}

(1) If there exist at least two disjoint $R(\tau)$ regular closed sets $F_{1}, F_{2}$. Then there exist disjoint $R(\tau)$ open sets $U$ and $V$ such that $F_{1} \subset U$ and $F_{2} \subset V$. Hence $Y=Y-(U \cap V)=(Y-U) \cup(Y-V)=\left(Y-F_{1}\right) \cup\left(Y-F_{2}\right)$. But $\left(Y-F_{1}\right)$ and $\left(Y-F_{2}\right)$ are $R S$-compact subspace. [Theorem 1.3 and Theorem 2.1]. By Proposition 2.1 and Proposition 2.2, we arrive $(Y, \tau)$ is $R S$-compact.

(2) If there do not exist two disjoint $R(\tau)$ regular closed sets. We assume that $F_{1}, F_{2} \in \mathbb{R} C(Y, R(\tau))$ such that $\phi \neq F_{1} \cap F_{2}=F$. By Lemma 2.2, $F_{1}, F_{2} \in$ $R O(Y, R(\tau))$. Then $F \in R O(Y, R(\tau))$ and $F \cup\left(Y-F_{1}\right) \cup\left(Y-F_{2}\right)=F \cup(Y-$ $\left.\left(F_{1} \cap F_{2}\right)\right)=F \cup(Y-F)=Y$. Since $F,\left(Y-F_{1}\right),\left(Y-F_{2}\right)$ are $R S$-compact subspaces and belongs to the class of regular open sets in $(Y, \tau)$. Thus $(Y, \tau)$ is $R S$-compact.

A point $x$ of $X$ is said to be $\delta$-adherent point of a subset $S$ of $X$ if int $\operatorname{cl}(V) \cap S \neq \phi$ for every open neighborhood $V$ of $x$ in $X$. A subset $S$ is said to be $\delta$-closed [24] if $S$ contains all $\delta$-adherent points of $S$. In a regular space $\delta$-closure operation is identical with the closure operation [24].

Theorem 2.3. [22]. If $X$ is $R S$-compact and $A$ is $\delta$-closed in $X$, then $A$. is $R S$-compact relative to $X$.

Theorem 2.4. If $(Y, \tau)$ is regular, then $(Y, \tau)$ is $R S$-compact iff $\tau=R(\tau)$.

Proof. Let $\tau=R(\tau)$, then $(Y, \tau)$ is $R S$-compact. Conversely, let $U \in \tau$, then $(Y-U)$ is closed in $\tau$. But $(Y, \tau)$ is regular, then $(Y-U)$ is $\delta$-closed. But 
$(Y, \tau)$ is $R S$-compact, and by using Theorem $2.3,(Y-U)$ is $R S$-compact relative to $\tau$. Hence $U \in R(\tau)$, and $\tau \subset R(\tau)$. Thus $R(\tau)=\tau$.

Theorem 2.5. Let $(Y, \tau)$ be a space such that every open set is closed, then $(Y, \tau)$ is $R S$-compact iff $\tau=R(\tau)$.

Proof. Let $U \in \tau$, then $(Y-U)$ is open and closed and hence regular open set. But $(Y, \tau)$ is $R S$-compact, then $(Y-U)$ is $R S$-compact relative to $\tau$. Thus $U \in R(\tau)$.

Theorem 2.6. If $(Y, \tau)$ is connected, then $(Y, R(\tau))$ has the property that $c l_{R(\tau)} U=X$, for all $U$ in $R(\tau)$.

Proof. Let $U \in R(\tau)$, then $c l_{R(\tau)} U \in R(\tau),[(Y, R(\tau))$ is extremely disconnected]. Thus $c l_{R(\tau)} U$ is both open and closed in $R(\tau)$, and also in $\tau$. But $(Y, \tau)$ is connected, which implies that $c l_{R(\tau)} U=X$.

\section{Separation Properties}

The property of being $T_{1}$-space is expansive, that is if $(Y, \tau)$ is $T_{1}$ and $\tau \subset \tau^{\prime}$ then $\left(Y, \tau^{\prime}\right)$ is $T_{1}$ but it is not generally contractive. The following result proves the contractivity of $T_{1}$ property from $(Y, \tau)$ to $(Y, R(\tau))$.

Lemma 3.1. If $(Y, \tau)$ is $T_{1}$, then $(Y, R(\tau))$ is $T_{1}$.

Proof. Let $x$ be any point of $Y$, then $\{x\}$ is closed in $\tau$ and $R S$-compact in $(Y, \tau)$. Thus $Y-\{x\}$ is open in $\tau$ and $\{x\}$ is $R S$-compact. Hence $Y-\{x\}$ is open in $R(\tau)$. Thus $(Y, R(\tau))$ is $T_{1}$.

Theorem 3.1. If $(Y, \tau)$ is Hausdorff, then $(Y, R(\tau))$ is compact.

Proof. From Lemma (3) in [20], we have that $R(\tau) \subset c\left(\tau_{s}\right) \subset \tau_{s} \subset n(\tau) \subset$ $\tau$. Using Lemma (4) in [19] and Theorem 2.1 in [23], the result follows.

Lemma 3.2. If $(Y, R(\tau))$ is Hausdorff, then $(Y, R(\tau))$ is normal. 
Proof. By using Theorem 3.1, and Theorem 1.6, the proof is obvious.

Lemma 3.3. If $(Y, R(\tau))$ is semi- $T_{2}^{\prime}$, then $(Y, \tau)$ is $R S$-compact space.

Proof. Let $x, y \in X$ and $x \neq y$, then there exist $W_{1}, W_{2} \in R C(Y, R(\tau))$ such that $x \in W_{1}, y \in W_{2}$ and $W_{1} \cap W_{2}=\phi$. Then $Y=Y-\left(W_{1} \cap W_{2}\right)=$ $\left(Y-W_{1}\right) \cup\left(Y-W_{2}\right)$. But $(Y, R(\tau))$ is $R S$-compact, then $\left(Y-W_{1}\right)$ and $\left(Y-W_{2}\right)$ are $R S$-compact relative to $R(\tau)$. By using Theorem 1.4, we can prove that they are $R S$-compact relative to $\tau$. Thus $(Y, \tau)$ is $R S$-compact space.

Theorem 3.2. [18]. If $\tau$ and $\tau^{\prime}$ are two topologies on $X$, such that $\tau \subset \tau^{\prime}$, then $R O(X, \tau)=R O\left(X, \tau^{\prime}\right)$ iff $c l_{\tau} G=c l_{\tau^{\prime}} G$ for each $G \in \tau^{\prime} ;\left[c l_{\tau} G=c l_{\tau^{\prime}} G\right.$ is equivalent to int $t_{\tau} F=$ int $_{\tau^{\prime}} F$, for each $\left.F \in \tau^{\prime c}\right]$.

Theorem 3.3[7]. If $(X, \tau)$ is a space, then $A \in P O(X, \tau)$ iff $A=U \cap V$, $U \in R O(X, \tau), V$ is $\tau$-dense.

Theorem 3.4. If $(Y, R(\tau))$ is semi-T, then the following statements hold:

(i) $R O(Y, R(\tau))=R O(Y, \tau)$.

(ii) $P O(Y, \tau) \subset P O(Y, R(\tau))$.

(iii) $S O(Y, R(\tau)) \subset S O(Y, \tau)$.

(iv) $\alpha O(Y, R(\tau)) \subset \alpha O(Y, \tau)$.

Proof. (i) Let $U \in R O(Y, \tau)$, then $(Y-U) \in R C(Y, \tau)$. By Lemma 3.3, $(Y, \tau)$ is $R S$-compact, and hence extremely disconnected, thus $(Y-U) \in \tau$. By Theorem 1.3, $U$ is $R S$-compact relative to $\tau$. Then $(Y-U) \in R(\tau)$, and $U$ is closed in $R(\tau)$, and also in $\tau$. Thus $(Y-U) \in R O(Y, \tau)$ and also is $R S$-compact relative to $\tau$. Hence $U \in R(\tau)$. Since $U$ is open and closed in $R(\tau)$, then $U \in$ $R O(Y, R(\tau))$. Hence $R O(Y, \tau) \subset R O(Y, R(\tau))$. But $R O(Y, R(\tau)) \subset R O(Y, \tau)$. Thus $R O(Y, R(\tau))=R O(Y, \tau)$.

(ii) Let $A \in P O(Y, \tau)$, then by Theorem 3.3, $A=U \cap V$, where $U \in R O(Y, \tau)$ and $V$ is $\tau$-dense. Since $R O(Y, R(\tau))=R O(Y, \tau)$ and each $\tau$-dense is $R(\tau)$-dense. Thus $A \in P O(Y, R(\tau))$, and $P O(Y, \tau) \subset P O(Y, R(\tau))$. 
(iii) Let $A \in S O(Y, R(\tau))$, then there exists $G \in R(\tau)$ such that $G \subset A \subset$ $c l_{R(\tau)} G$. Since $R(\tau) \subset \tau$, then $G \in \tau$. But $R O(Y, R(\tau))=R O(Y, \tau)$, then by Theorem $3.2 c l_{R(\tau)} G=c l_{\tau} G$. Hence $A \in S O(Y, \tau)$.

(iv) Let $G \in \alpha O(Y, R(\tau))$, then $G \subset \operatorname{int}_{R} c l_{R} \operatorname{int}_{R} G \subset \operatorname{int}_{\tau} c l_{R} \operatorname{int}_{\tau} G=$ $\operatorname{int}_{\tau} c l_{\tau} \operatorname{int}_{\tau} G$ [by Theorem 3.2]. Hence $G \in \alpha O(Y, \tau)$.

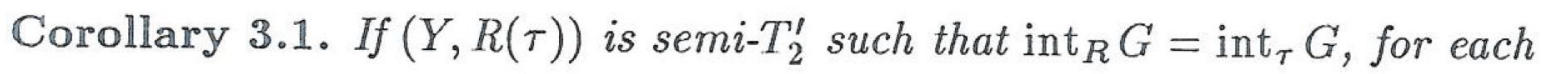
$G \in \alpha O(Y, \tau)$, then $\alpha O(Y, R(\tau))=\alpha O(Y, \tau)$.

Proof. If $G \in \alpha O(Y, \tau)$, then $G \subset \operatorname{int}_{\tau} c l_{\tau} \operatorname{int}_{\tau} G=\operatorname{int}_{R} c l_{\tau} \operatorname{int}_{R} G=\operatorname{int}_{R}$ $c l_{R} \operatorname{int}_{R} G$. [By Theorem 3.2].

Corollary 3.2. If $(Y, R(\tau))$ is semi- $T_{2}^{\prime}$, and $(Y, \tau)$ is $\alpha$-compact, then $(Y, R(\tau))$ is $\alpha$-compact.

Proof. By Theorem 3.4(iv).

Corollary 3.3. If $(Y, R(\tau))$ is semi- $T_{2}^{\prime}$, and $(Y, R(\tau))$ is strongly compact, then $(Y, \tau)$ is strongly compact.

Proof. By Theorem 3.4 (ii).

Theorem 3.5. $(Y, R(\tau))$ is semi- $T_{2}$ iff $(Y, R(\tau))$ is semi- $T_{2}^{\prime}$.

Proof. Let $(Y, R(\tau))$ be semi- $T_{2}$, and $x, y \in Y, x \neq y$, then there exist $U, V \in S O(Y, \tau)$ such that $x \in U, y \in V$ and $U \cap V=\phi$, which implies that $c l_{R}(U \cap V)=\phi$. Since $(Y, R(\tau))$ is extremely disconnected we have $c l_{R} \operatorname{int}_{R} U=$ $c l_{R} U \in \tau$, and $c l_{R} \operatorname{int}_{R} V=c l_{R} V \in \tau$. But $c l_{R} U \cap c l_{R} V=c l_{R}(U \cap V)=\phi$. Hence $(Y, R(\tau))$ is semi- $T_{2}^{\prime}$. The converse is obvious.

Theorem 3.6. $(Y, R(\tau))$ is Hausdorff iff $(Y, R(\tau))$ is semi- $T_{2}$.

Proof. It is similar to the proof of Theorem 3.5.

Theorem 3.7. Let $(Y, \tau)$ be a space, then:

a) The class of $S O(Y, R(\tau))$ form a topology (denoted by $\left.\tau^{\prime}\right)$ finer than $R(\tau)$. 
b) $R O(Y, R(\tau))=R O\left(Y, \tau^{\prime}\right)$.

c) $\left(Y, \tau^{\prime}\right)$ is $R S$-compact.

Proof. (a) Since $(Y, R(\tau))$ is $R S$-compact, it is extremely disconnected and hence $S O(Y, R(\tau))$ forms a topology such that $R(\tau) \subset \tau^{\prime}$.

(b) Let $G \in \tau^{\prime}$, then $c l_{\tau^{\prime}} G \subset c l_{R} G$. Conversely, let $x \in c l_{R} G$, and $x \in U$, $U \in \tau^{\prime}$. Hence $x \in c l_{R} \operatorname{int}_{R} U \in R(\tau)$ and $c l_{R} \operatorname{int}_{R} U \cap G \neq \phi$. But $G \in \tau^{\prime}$, implies $G \in S O(Y, R(\tau))$ and $G \subset c l_{R} \operatorname{int}_{R} G$, therefore $\phi \neq c l_{R} \operatorname{int}_{R} G \cap c l_{R} \operatorname{int}_{R} U \subset$ $c l_{R}\left(\operatorname{int}_{R} G \cap c l_{R} \operatorname{int}_{R} U\right) \subset c l_{R}\left(\operatorname{int}_{R} G \cap i n t_{R} U\right) \subset c l_{R}(U \cap G)$, which implies that $U \cap G \neq \phi$, and so $x \in c l_{\tau^{\prime}} G$. Hence $c l_{R} G \subset c l_{\tau^{\prime}} G$. Thus $c l_{R} G=c l_{\tau^{\prime}} G$ for each $G \in \tau^{\prime}$.

(c) Using (b), the result follows.

Lemma 3.4. If $(Y, \tau)$ is $R_{0}$, then $(Y, R(\tau))$ is $R_{0}$.

Proof. Let $x \in Y$, and $x \in G \in R(\tau)$, then $G \in \tau$, and $c l_{\tau}\{x\} \subset G$. Since $c l_{\tau}\{x\}$ is compact in $(Y, \tau)$, implies that it is compact in $(Y, R(\tau))$, and nearly compact in $(Y, R(\tau))$. But $(Y, R(\tau))$ is extremely disconnected, then $c l_{\tau}\{x\}$ is $R S$-compact relative to $R(\tau)$, which implies that it is $R S$-compact relative to $\tau$. Thus $c l_{\tau}\{x\}$ is closed in $(Y, R(\tau))$, implies that $c l_{R}\{x\} \subset c l_{\tau}\{x\}$. Hence $c l_{R}\{x\}=c l_{\tau}\{x\}$, and $(Y, R(\tau))$ is $R_{0}$.

Theorem 3.8. Minimal $R_{0}$-spaces are $R S$-compact spaces.

Proof. Using Lemma 3.4, the result follows.

\section{Acknowledgement}

We would like to thank the referee for valuable comments and suggestions.

\section{References}

[1] M. E. Abd El-Monsef, "Studies on some pretopological concepts", Ph. D. Thesis, Tanta University (1980).

[2] M. E. Abd El-Monsef, R. A. Mahmoud and A. A. Nasef, "Functions based on compactness", (to appear). 
[3] R. H. Atia, S. N. El-deeb and A. S. Mashhour, " $\alpha$-compactness and $\alpha$-homeomorphism", (preprint).

[4] D. E. Cameron, "Properties of S-closed spaces", Proc. Amer. Math. Soc., 72 (3) (1978), 581-585.

[5] —_ "Some maximal topologies which are QHC" Proc. Amer. Math. Soc., 75 (1) (1979), 149-156.

[6] A. S. Davis, "Indexed systems of neighborhoods for general topological spaces", Amer. Math. Month., 68 (1961), 886-893.

[7] M. Ganster, "Preopen sets and resolvable spaces", to appear in Kyungpook Math. J.

[8] D. B. Gauld, M. Mrsevic, I. L. Reilly, and M. K. Vamanamurthy, "Colindelof topologies and $L$-continuous functions", Clasnik, Math., 19 (39) (1984), 297-308.

[9] W. C. Hong, "RS-compact spaces", J. Korean. Math. Soc., 17 (1980), 39-43.

[10] S. T. Hu, "Elements of general topology", Holden Day, IM, (1972).

[11] D. S. Jankovic, "On locally irreducible spaces", Ann. de la Soc. Sci de Bruxelles, T, 97, II, (1983), 59-72.

[12] A. M. Kozae, "Studies on some maximal and minimal topological concepts", $P h . D$. Thesis, Tanta University (1988).

[13] N. Levine, "Semi-open sets and semi continuity in topological spaces", Amer. Math. Monthly 70 (1963), 36-41.

[14] S. N. Maheshwari and R. Prasad, "Some new separation axioms", Ann. Soc. Sci. Bruxelles, T. 3 (89) (1975), 395-407, MR 52 \# 6660.

[15] A. S. Mashhour, M. E. Abd El-Monsef and S. N. El-Deeb, "On pretopological spaces", Bubl. Math. de la Soc. R. S. de Roumanie 28 (76) (1984), 39-45.

[16] A. S. Mashhour, M. E. Abd El-Monsef, I. A. Hasanein and T. Noiri, "Strongly compact spaces", Delta J. Sci. 8 (1) (1984), 30-46.

[17] A. S. Mashhour, F. S. Mahmoud, I. A. Hasanein and M. A. Fath Alla, "On some generalization of compactness", (to appear).

[18] J. Mioduszewski and L. Rudolf, " $H$-closed and extremely disconnected spaces", Dissertations Math., 66 (1969).

[19] M. Mrsevic, I. L. Reilly and M. K. Vamanamurthy, "On semi-regularization topologies", J. Austral. Math. Soc., (Series A) 38 (1985), 40-54.

[20] M. Mrsevic and I. L. Reilly, "On $N$-continuity and Co- $N$-closed topologies", Ricerche di Math., 36 (1) (1987), 33-43.

[21] O. Njasted, "On some classes of nearly open sets", Pac. J. of Math. 15 (3) (1965), 961-970.

[22] T. Noiri, "On RS-compact spaces", J. Korean Math. Soc., 22 (1985), No. 1, pp. 19-34.

[23] M. K. Singal and A. Mathur, "On nearly compact spaces II", Boll, Della Un. Math. Italiana, (4) 9 (1974), 670-678.

[24] N. V. Velicko, "H-closed topological spaces", Amer. Math. Soc. Transl., (2) 78 (1968), 103-118.

Department of Mathematics, Faculty of Science, Tanta University, Tanta, Egypt. 